Radial and Nonradial Pulsations as Probes of Stellar Physics

ASP Conference Series, Vol. 259, 2002

C. Aerts, T.R. Bedding, \& J. Christensen-Dalsgaard, eds.

\title{
Pulsating AGB Stars in the LMC
}

\author{
Jacco Th. van Loon \\ Astrophysics Group, School of Chemistry 8 Physics, Keele University, \\ Staffordshire ST5 5BG, United Kingdom (jacco@astro.keele.ac.uk)
}

Abstract. I give a brief review and interpretation of the evolution, mass loss and pulsation of AGB stars in the Large Magellanic Cloud.

\section{The Magellanic Clouds in an astro-physical context}

Our view of the place of mankind in the Universe changed dramatically with the recognition by Abbe (1967) that the Large and Small Magellanic Clouds (LMC \& SMC) are in fact nearby galaxies composed of stars and nebular matter, separate from our own Milky Way galaxy. Four decades later, the discovery in 1907 by Henrietta Leavitt of a relation between the pulsation period and apparent brightness of Cepheid-type variables in the LMC counts amongst the most important discoveries in modern astronomy.

Over the past few decades, a great deal of astro-physics has been learnt from studies of stars in the Magellanic Clouds, for several reasons: (i) stars in each of the Clouds are all at the same distance to us, and therefore their relative luminosities are well determined; (ii) this distance is well known; (iii) the Clouds are near enough to study individual stars; (iv) interstellar extinction to and within the Clouds is small; (v) as dwarf galaxies, the Clouds are fairly big, and they have experienced star formation over much of their history. Hence, plenty of stars can be found in different evolutionary stages, including stars in clusters for which accurate ages may be determined. (vi) The LMC and SMC have metallicities of $\sim \frac{1}{2}$ and $\frac{1}{5}$ solar, allowing the study of metallicity effects. However, as the analyses and astrophysical problems they address become more detailed, these advantages meet their limitations: (i) the depth of the Clouds has been measured and is not always negligible; (ii) different methods yield a distance modulus of the LMC ranging from $\sim 18.1$ to $18.7 \mathrm{mag}$ (I adopt distance moduli to the LMC and SMC of 18.5 and $19.0 \mathrm{mag}$, respectively); (iii) current instrumentation does not always provide the required spatial resolution or detection sensitivity; (iv) although extinction is much less than in the plane of the Milky Way, it is not always negligible even at infrared (IR) wavelengths, and can be very patchy in places; (v) the number of stars in very rare, short-lived evolutionary phases is limited - sometimes to less than one at any moment - hampering statistical studies. Also, the star formation history of the LMC is complex, with a lack of 4 to 10 Gyr-old stellar clusters. And (vi) there are no (identified) samples of either metal-rich stars, with $[\mathrm{Fe} / \mathrm{H}]>0$, or extremely metal-poor stars, with $[\mathrm{Fe} / \mathrm{H}] \ll-2$. 


\section{Asymptotic Giant Branch (AGB) stars}

The AGB phase is the final stage in the life of a star with a Zero-Age MainSequence (ZAMS) mass of $\sim 0.5$ to $8 \mathrm{M}_{\odot}$. The lower limit to this mass range is set by the requirement that the temperature and pressure at the bottom of the hydrogen mantle surrounding the electron-degenerate core (oxygen and carbon/neon) is sufficiently high to ignite hydrogen shell burning, driving the evolution of the star along the asymptotic giant branch towards higher luminosity (growing core) and lower surface temperature (expanding mantle). The AGB evolution terminates either because the core has reached the Chandrasekhar mass limit of $M_{\max } \sim 1.4 \mathrm{M}_{\odot}$ beyond which the core becomes gravitationally unstable, the corresponding luminosity of $L_{\max } \sim 55,000 \mathrm{~L}_{\odot}$ defining the tip of the AGB, or because the mantle has diminished to the extent that the hydrogenburning shell becomes exposed and quickly runs out of fuel, leaving the hot and compact core (white dwarf) to cool into oblivion. The upper limit to the mass range of $\mathrm{AGB}$ progenitors is set by the condition for the ignition of carbon burning in the non-degenerate core of supergiants.

The hydrogen-burning shell produces an underlying helium shell which ignites as soon as sufficient pressure has built up, thereby causing the mantle to expand and the hydrogen burning to switch off. Such Thermal Pulses (TP) re-occur on timescales from $\Delta t \sim 10^{3}$ yr for the most massive AGB stars up to $\Delta t \sim 10^{5} \mathrm{yr}$ for the least massive AGB stars. The TP itself is brief $(\sim 1 \%$ of $\Delta t)$, but it takes long (up to $\sim 30 \%$ of $\Delta t$ ) for a star to recover from the luminosity dip of up to a factor two whilst the hydrogen shell burning gradually takes over from the helium shell burning. The effect of the TP on the mantle and emergent luminosity is relatively stronger for less massive stars. The TP-AGB phase lasts for $t=$ few $\times 10^{5} \mathrm{yr}$, up to $t=$ few $\times 10^{6} \mathrm{yr}$ for the most massive AGB stars unless mass loss is severe.

Energy transport from the nuclear burning site to the stellar surface of an AGB star proceeds via convective motion through the mantle: a rising gas element continues to rise. Much theoretical understanding needs to be gained about the exact criterion for convective instability and the description of convective flows and boundaries, especially because convection not only transports energy but also matter and hence affects the stratification of elemental abundances throughout the mantle. When during a TP the convection zone penetrates into layers containing the products of nuclear burning, these heavy elements will become mixed into and become over-abundant in the stellar photosphere where light leaves the star. This is the third time in the evolution of intermediate-mass stars that such dredge-up event may happen, and probably occurs only for the more massive AGB stars $\left(M \gtrsim 1.5 \mathrm{M}_{\odot}\right)$. Products of the slow neutron-capture process, s-process elements such as zirconium, technetium and barium are used to diagnose the TP-AGB history of stars. Carbon may become enhanced too, greatly affecting the chemistry in the cool stellar atmosphere $\left(T_{\text {eff }} \lesssim 4000 \mathrm{~K}\right)$ if, after binding carbon and oxygen into carbon-monoxide (CO), not oxygen but carbon remains to form more complex molecules. Such carbon stars are easily distinguished from oxygen-rich AGB stars as their spectra are dominated by absorption bands of $\mathrm{CN}, \mathrm{HCN}, \mathrm{C}_{2}$ and $\mathrm{C}_{2} \mathrm{H}_{2}$ rather than $\mathrm{TiO}, \mathrm{VO}$ and $\mathrm{SiO}$. However, nuclear burning may take place at the inner boundary of the convection zone, with this Hot Bottom Burning (HBB) converting carbon into oxygen 
and nitrogen and preventing the formation of a carbon star. HBB occurs in massive AGB stars $\left(M \gtrsim 4 \mathrm{M}_{\odot}\right)$ and enhances their luminosity, but switches off once the mantle has nearly gone due to severe mass loss.

The stability criterion for motion within the mantle not only determines the occurrence of convection (turbulent motion), but also the occurrence of pulsation (organised motion). In the hydrogen ionization zone not far below the stellar surface, and in the first helium ionization zone, the absorption coefficient of the gas to radiation from the stellar interior is very large; hence the internal energy becomes high and the mantle expands until the opacity drops and the mantle shrinks again. These radial pulsations levitate the atmosphere high enough for dust formation to occur and radiation pressure to blow away the circumstellar dust - taking with it the collisionally coupled gas. Mass-loss rates of $\dot{M} \sim 10^{-7}$ to $10^{-4} \mathrm{M}_{\odot} \mathrm{yr}^{-1}$ cause the premature termination of AGB evolution, exposing the white dwarf after the star has shed its mantle.

\section{AGB stars in the Magellanic Clouds}

Searches for AGB stars in the Magellanic Clouds have followed three main strategies: (i) monitoring surveys to find Long-Period Variables (LPVs), mostly of Mira type, defined by regular pulsation on the order of a year with a visual amplitude $A_{\mathrm{V}} \geq 2.5 \mathrm{mag}$; (ii) spectroscopic or photometric surveys to find bright M-type or carbon stars, and (iii) mid-IR surveys to find AGB stars with circumstellar dust emission.

\subsection{Optically bright AGB stars}

Amongst the most influential systematic searches for magellanic M-type giants and carbon stars are the study of selected fields in the bar of the LMC and in the SMC (Blanco et al., 1980; Blanco \& McCarthy, 1983; Frogel \& Richer, 1983), the construction of the carbon star luminosity function in the LMC (Costa \& Frogel, 1996), and the survey of magellanic clusters for which ages and hence the ZAMS masses of the cluster members can be determined (Aaronson \& Mould, 1985; Frogel et al., 1990; Westerlund et al., 1991).

Although much of the physics remains in parameterised form - notably convection and dredge-up - evolutionary models can be tuned to reproduce the carbon star luminosity function quite well (Marigo et al., 1999). When these results are confronted with the luminosities and chemistry of cluster AGB stars in the Magellanic Clouds (Frogel et al., 1990), the match is not entirely satisfactory (Fig. 1, left), due to both imperfections in the models as well as difficulties in deducing the observed quantities.

The first grand survey for variable stars resulted in the catalogue of Harvard Variables (Payne-Gaposchkin, 1971) which, however, were mostly supergiants. Subsequent surveys identified pulsating AGB stars in sufficient numbers for a P-L relation to be proposed (Glass \& Lloyd-Evans, 1981) and refined (Wood et al., 1983; Feast et al., 1989; Hughes \& Wood, 1990). Miras did indeed seem to obey a remarkably tight and unique P-L relation (Fig. 1, right) suggesting a robust instability threshold and a single pulsation mode, and their possible use as distance indicators, but already a few complications may be noticed: (i) The P-L relation has a different slope for periods shorter/longer than $P^{\prime} \sim 400 \mathrm{~d}$, which 

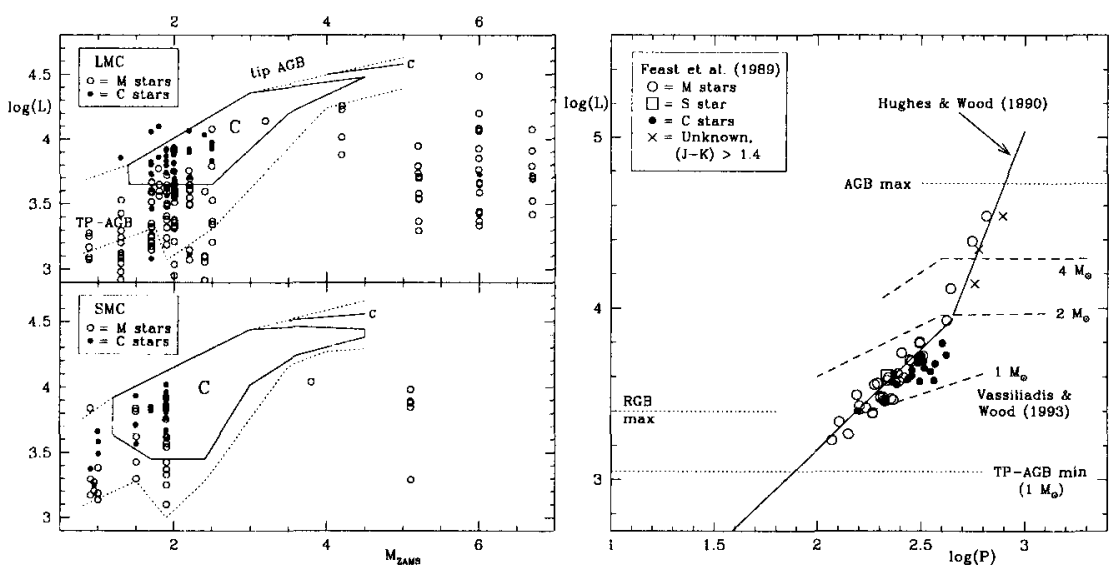

Figure 1. Left: models (Marigo et al., 1999) and cluster data (Frogel et al., 1990); Right: P-L relation in the LMC (Feast et al., 1989; Hughes \& Wood, 1990) and evolutionary tracks (Vassiliadis \& Wood, 1993).

evolutionary tracks (Vassiliadis \& Wood, 1993) link to a ZAMS mass $M^{\prime} \sim 2$ $\mathrm{M}_{\odot}$. Previous evolution, for instance whether $\left(M<M^{\prime}\right)$ or not $\left(M>M^{\prime}\right)$ the star experienced violent ignition of core-helium burning at the tip of the first red giant branch (RGB), may be the cause for a difference in internal structure and pulsational properties. Also, (ii) carbon stars and especially red stars have somewhat longer periods for their luminosities, which may be due to their larger radii, as they have suffered significant mass loss. (iii) A paucity of luminous AGB stars, in particular carbon stars with $L \gtrsim 20,000 \mathrm{~L}_{\odot}$ corresponding to $M \gtrsim 4 \mathrm{M}_{\odot}$, was explained as due to HBB (Iben, 1981; Wood et al., 1983). This was confirmed by the identification of lithium-enriched S-type stars $(\mathrm{C} / \mathrm{O}$ just below unity) with $20,000<L<55,000 \mathrm{~L}_{\odot}$ (Smith et al., 1995).

\subsection{Dust-enshrouded AGB stars}

Studies of optically bright AGB stars are biased against stars in the final phases of AGB evolution that have become dust-enshrouded as a result of mass loss. It is difficult to study their properties as their stellar surface is obscured at optical wavelengths and the photosphere at IR wavelengths becomes dominated by thermal emission from the circumstellar dust.

The IRAS mission in the early 1980s provided a catalogue with a few hundred mid-IR point sources in the Magellanic Clouds that are candidate dust shells around AGB stars (Loup et al., 1997). Follow-up on such IRAS sources and similar dust-enshrouded supergiants (Wood et al., 1992; van Loon et al., 1998 , and references therein) culminated in the detailed quantitative study of $\sim 50$ dust-enshrouded AGB stars in the LMC observed with ISO (van Loon et al., 1999). The main results are that (i) luminous carbon stars do exist amongst dust-enshrouded AGB stars, confirming the HBB scenario with mass loss; (ii) 

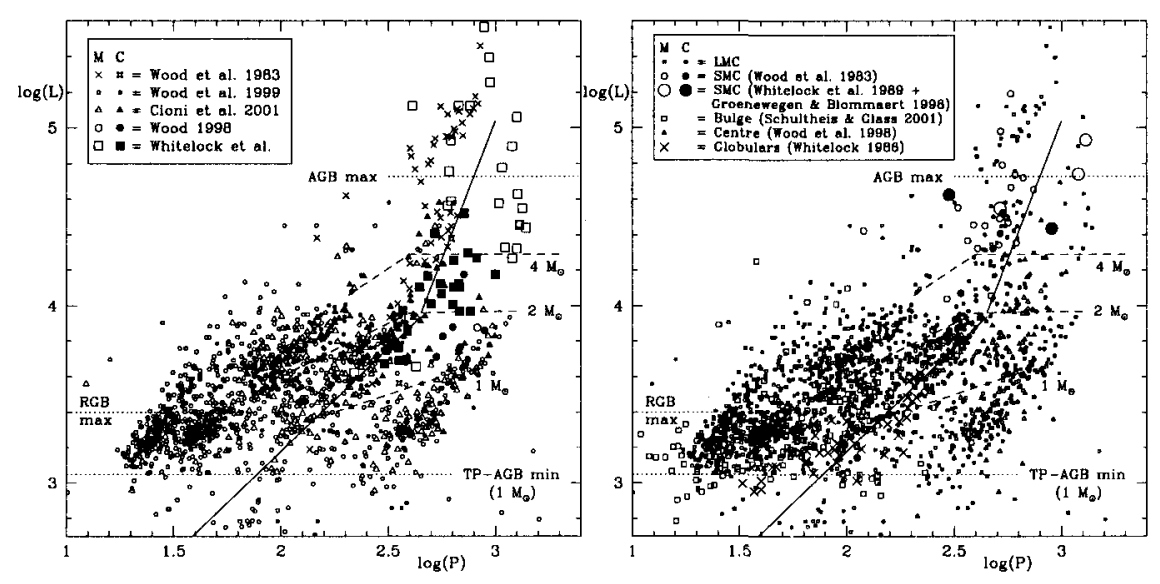

Figure 2. P-L diagram in the LMC (left), and compared with the $\mathrm{SMC}$ and the galactic bulge, centre and globular clusters (right).

the threshold above which HBB occurs is confirmed to be $4 \mathrm{M}_{\odot}$ (van Loon et al., 2001); (iii) winds of AGB stars are dust-driven (e.g. Gail \& Sedlmayr, 1986); (iv) mass-loss rates exceed nuclear burning mass consumption rates and reach a maximum $\dot{M}_{\max } \propto L$ in a distinct superwind phase; (iv) mass-loss rates increase with lower $T_{\text {eff }}$ - this effect is strongest during the early stages of circumstellar dust formation - but may be only slightly dependent on metallicity for $[\mathrm{Fe} / \mathrm{H}] \gtrsim-1$ (van Loon, 2000). Great improvement in our knowledge of AGB mass loss is expected from recent surveys of the Magellanic Clouds at near-IR (2MASS, DENIS) and mid-IR (MSX, ISO) wavelengths, and their follow-up.

\subsection{Large-scale variability surveys}

Much progress is made in detecting variables in the LMC thanks to micro-lensing surveys (MACHO, EROS, OGLE). The P-L diagram (Fig. 2, left) not only shows the classical Mira sequence but at least three more roughly parallel sequences. Two of these, at shorter periods and populated by semi-regulars (many of which are regular but have smaller amplitudes than Miras), are believed to correspond to overtone modes with the Miras pulsating in the fundamental mode (Wood \& Sebo, 1996; Wood et al., 1999). This interpretation is supported by the behaviour of pulsation amplitudes \& velocities (Scholz \& Wood, 2000), period $\&$ radius, and non-linear models (Ya'ari \& Tuchman, 1996, 1999) for galactic Miras, but if the mixing length describing convection is allowed to vary then Miras could still pulsate in the first overtone (Barthès, 1998). Another sequence at longer periods than the Mira P-L is explained as binarity (Wood et al., 1999).

It is estimated that not all but $\sim 65 \%$ of the TP-AGB stars are LPV, of which (only) $\sim 10 \%$ are Miras (Cioni et al., 2001), and the evolution in the $\mathrm{P}-\mathrm{L}$ plane is complex: the pulsation is a function of mass and time - not 
necessarily in a continuous or even monotonic fashion. It is then remarkable how consistently the P-L diagram is populated by stars in the LMC, SMC and galactic centre, and the galactic bulge $\left(M \lesssim 2 \mathrm{M}_{\odot}\right)$ and globular clusters $(M \lesssim 1$ $\mathrm{M}_{\odot}$ ), despite an order of magnitude difference in metallicity (Fig. 2, right).

An overdensity of stars at the two short-period sequences just below the maximum luminosity at the RGB indicates these may be RGB stars, whilst above the maximum luminosity at the AGB, supergiants are found on sequences similar to but slightly displaced from the Mira P-L and the long-period sequence. This suggests that certain similarities in the internal structure of RGB stars, AGB stars and (red) supergiants cause similarities in their pulsation.

Carbon stars populate the Mira P-L where stars with $M_{\text {ZAMS }} \sim 1.5$ to 4 $\mathrm{M}_{\odot}$ are found, as expected from third dredge-up and HBB. Carbon stars on the sequence immediately shortward in period from the Mira P-L, at $L \sim 8,000 \mathrm{~L}_{\odot}$, may have $M_{\text {ZAMS }} \sim 3$ to $4 \mathrm{M}_{\odot}$, but the continuation of the evolutionary track is devoid of stars until it reaches the Mira P-L at twice this luminosity. Perhaps, after having become carbon stars on the Mira P-L, these stars are temporarily semi-regulars, possibly due to a TP. Surprisingly, the most luminous carbon stars obey the Mira P-L. Thought to have become carbon-enriched after an extended period of severe mass loss terminated HBB, they would be expected to have the longest periods of all - longer than on the Mira P-L.

\section{The connection between pulsation and mass loss of AGB stars}

When AGB stars arrive on the Mira P-L, their mass-loss rates have become so severe that further evolution is along tracks of constant luminosity, with the radius (and hence pulsation period) growing as the structure of the mantle readjusts itself to the decrease in mass. Hence the dust-enshrouded stars (Wood, 1998; Whitelock et al., in preparation) are on the Mira P-L or to longer periods. Describing the Mira as a polytrope $(R \sqrt[3]{M}=$ constant $)$ and the pulsation as a harmonic oscillator $\left(2 \pi / P \simeq 1 / t_{\text {freefall }} \simeq g(R) / R\right)$, the period evolves as $P \propto 1 / M$. For instance, a Mira with $P=250 \mathrm{~d}\left(M_{\text {ZAMS }}=1.5 \mathrm{M}_{\odot}\right)$ would end up on the long-period sequence at $P \sim 750 \mathrm{~d}$ and $M \sim 0.5 \mathrm{M}_{\odot}$, which is a reasonable white dwarf mass. The longest observed period for an evolved equivalent of a Mira with $P=700 \mathrm{~d}\left(M_{\text {ZAMS }}=5 \mathrm{M}_{\odot}\right)$, however, has $P \sim 1400 \mathrm{~d}$ and $M \sim 2.5 \mathrm{M}_{\odot}$. Such star must still lose more than a solar mass, but stars with correspondingly long periods $(P \sim 3000 \mathrm{~d})$ have not (yet) been found.

The growth in radius/period of mass-losing AGB stars may become truncated when the dynamical timescale, $P$, approaches the thermal timescale, $t \simeq G M^{2} / L R$, and the mantle no longer pulsates adiabatically. This happens when $P \simeq \sqrt[3]{\pi M / \sigma T_{\text {eff }}^{4}}$, corresponding to $P \sim 2000 \mathrm{~d}$ for a star of $M=2.5 \mathrm{M}_{\odot}$ and $T_{\text {eff }}=2500 \mathrm{~K}$ (note the moderate dependence on stellar parameters). By this time, the surface gravity is so low that mass loss could be initiated without the help of pulsation: the star may quite literally be boiling over, and this could be responsible for the superwind phase required to shed the remainder of the mantle that may become visible as a planetary nebula. The ability of the mantle to restore thermo-dynamic equilibrium on a dynamical timescale may also be relevant for the faint part of the long-period sequence $\left(L \lesssim 10,000 \mathrm{~L}_{\odot}\right)$. Some 

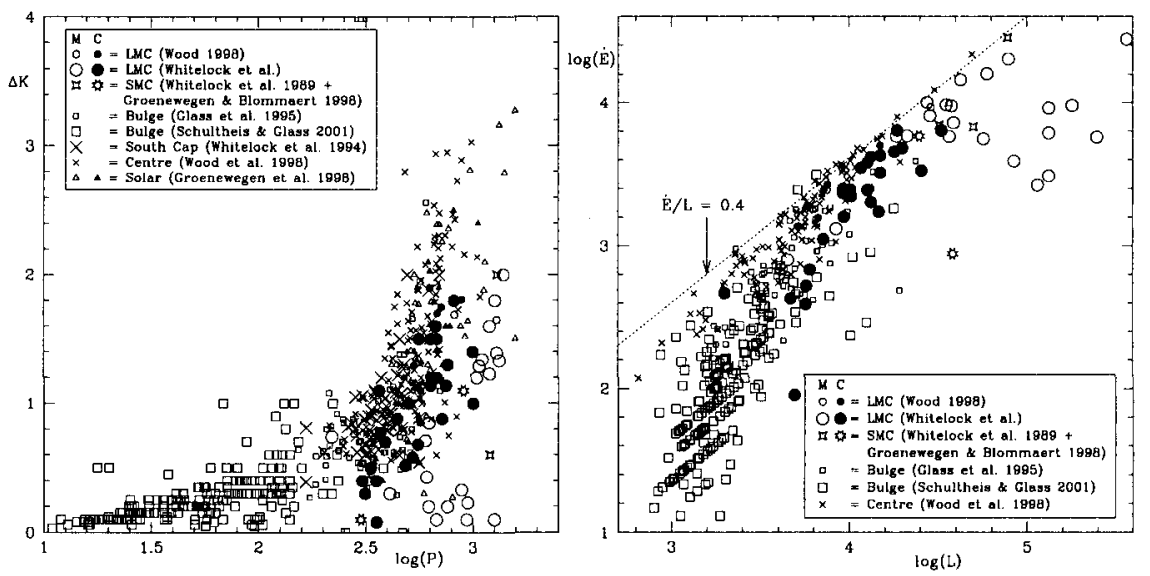

Figure 3. K-band amplitudes and pulsation energy input rate, versus period (left) and luminosity (right) in the LMC, SMC and Milky Way.

disturbance, for instance tidal interaction in a binary, could trigger instabilities on a thermal (not orbital) timescale, causing irregular mass loss that results in obscuration events, super-imposed on a "traditional" (adiabatic) pulsation in the fundamental mode (Mira P-L) or overtone mode (shorter-period sequences).

K-band amplitudes (a measure for the bolometric amplitude) generally increase with period, but for $P \gtrsim 400 \mathrm{~d}$ the increase becomes steeper (Fig. 3, left). This could be related to the different slope of the Mira P-L relation for periods shorter/longer than $P^{\prime} \sim 400 \mathrm{~d}$. The diagram suggests that, for longer periods, carbon stars and stars in the Magellanic Clouds pulsate less vigorously than galactic oxygen-rich stars. However, the pulsation of a luminous star with small amplitude may well involve more energy than that of a faint star with large amplitude. The time-averaged rate at which radiative energy is stored in the mechanical pulsation, $\dot{E}=L / 2 \times(\exp [\Delta K / 2.5]-1) /(\exp [\Delta K / 2.5]+1)$ has a theoretical maximum of $\dot{E}_{\max } / L=0.5$ : the maximum energy rate available during that half of the pulsation cycle in which the mantle is lifted is $L$. The observed $\dot{E}$ increases with luminosity (Fig. 3 , right), saturating at $\dot{E}_{\max } / L=0.4$ - i.e. very efficient - with no clear distinction between stars of different chemistry. As the maximum mass-loss rate was found to increase proportionally to luminosity (van Loon et al., 1999), I obtain $\dot{M}_{\max } \propto \dot{E}_{\max }$. This explains the absence of a clear metallicity-dependence of the mass-loss rate (van Loon 2000).

\section{References}

Aaronson M., Mould J., 1985, ApJ, 288, 551

Abbe C., 1867, MNRAS, 27, 262 
Barthès D., 1998, A\&A, 333, 647

Blanco V.M., McCarthy M.F., 1983, AJ, 88, 1442

Blanco V.M., Blanco B.M., McCarthy M.F., 1980, ApJ, 242, 938

Cioni M.-R.L., et al., 2001, A\&A, in press

Costa E., Frogel J.A., 1996, AJ, 112, 2607

Feast M.W., et al., 1989, MNRAS, 241, 375

Frogel J.A., Richer H.B., 1983, ApJ, 275, 84

Frogel J.A., Mould J., Blanco V.M., 1990, ApJ, 352, 96

Gail H.-P., Sedlmayr E., 1986, A\&A, 161, 201

Glass I.S., Lloyd-Evans T.L., 1981, Nature, 291, 303

Glass I.S., et al., 1995, MNRAS, 273, 383

Groenewegen M.A.T., Blommaert J.A.D.L., 1998, A\&A, 332, 25

Hughes S.M.G., Wood P.R., 1990, AJ, 99, 784

Iben I., 1981, ApJ, 246, 278

Loup C., et al., 1997, A\&AS, 125, 419

Marigo P., Girardi L., Bressan A., 1999, A\&A, 344, 123

Payne-Gaposchkin C.H., 1971, The variable stars of the Large Magellanic Cloud

Scholz M., Wood P.R., 2000, A\&A, 362, 1065

Schultheis M., Glass I.S., 2001, MNRAS, in press

Smith V.V., et al., 1995, ApJ, 441, 735

van Loon J.Th., 2000, A\&A, 354, 125

van Loon J.Th., et al., 1998, A\&A, 329, 169

van Loon J.Th., et al., 1999, A\&A, 351, 559

van Loon J.Th., et al., 2001, A\&A, 368, 950

Vassiliadis E., Wood P.R., 1993, ApJ, 413, 641

Westerlund B.E., et al., 1991, A\&AS, 91, 425

Whitelock P.A., 1986, MNRAS, 219, 525

Whitelock P.A., et al., 1989, MNRAS, 238, 769

Whitelock P.A., et al., 1994, MNRAS, 267, 711

Wood P.R., 1998, A\&A, 338, 592

Wood P.R., Sebo K.M., 1996, MNRAS, 282, 958

Wood P.R., Bessell M.S., Fox M.W., 1983, ApJ, 272, 99

Wood P.R., et al., 1992, ApJ, 397, 552

Wood P.R., Habing H.J., McGregor P.J., 1998, A\&A, 336, 925

Wood P.R., et al., 1999, in: IAU Symposium 191, p151

Ya'ari A. Tuchman Y., 1996, ApJ, 456, 350

Ya'ari A. Tuchman Y., 1999, ApJ, 514, L35 